\title{
Novel insight into the function of tankyrase (Review)
}

\author{
MI KYUNG KIM \\ Department of Systems Biology, College of Life Science and Biotechnology, \\ Yonsei University, Seoul 03722, Republic of Korea
}

Received May 15, 2018; Accepted August 31, 2018

DOI: $10.3892 / \mathrm{ol} .2018 .9551$

\begin{abstract}
Tankyrases are multifunctional poly(ADP-ribose) polymerases that regulate a variety of cellular processes, including Wnt signaling, telomere maintenance and mitosis regulation. Tankyrases interact with target proteins and regulate their interactions and stability through poly(ADP-ribosyl) ation. In addition to their roles in telomere maintenance and regulation of mitosis, tankyrase proteins regulate tumor suppressors, including AXIN, phosphatase and tensin homolog and angiomotin. Therefore, tankyrases may be effective targets for cancer treatment. Tankyrase inhibitors could affect a variety of carcinogenic pathways that promote uncontrolled proliferation, including Wnt, AKT, yes-associated protein, telomere maintenance and mitosis regulation. Recently, novel aspects of the function and mechanism of tankyrases have been reported, and a number of tankyrase inhibitors have been identified. A combination of conventional chemotherapy agents with tankyrase inhibitors may have synergistic anticancer effects. Therefore, it is expected that more advanced and improved tankyrase inhibitors will be developed, enabling novel therapeutic strategies against cancer and other tankyrase-associated diseases. The present review discusses tankyrase function and the role of tankyrase inhibitors in the treatment of cancer.
\end{abstract}

\section{Contents}

1. Introduction

2. Post-translational modification and tankyrase activity

3. Tankyrase and cancer

4. Tankyrase inhibitors

5. Novel tankyrase binding partners

6. Conclusions

Correspondence to: Dr Mi Kyung Kim, Department of Systems Biology, College of Life Science and Biotechnology, Yonsei University, 50 Yonsei-ro, Seoul 03722, Republic of Korea

E-mail: biokyung@gmail.com

Key words: tankyrase, post-translational modification, tankyrase inhibitors, cancer therapy, novel tankyrase binding partners

\section{Introduction}

Poly(ADP-ribose) polymerases (PARPs) are a large protein family involved in various cellular and molecular processes (1-8). PARPs transfer ADP-ribose molecules from donor $\mathrm{NAD}^{+}$to target proteins by post-translational modification, including poly(ADP-ribosyl)ation (PARsylation) (1-3). PARsylation regulates numerous cellular processes, including DNA damage repair (4), cellular stress signaling (5), gene transcription $(6,7)$ and ageing (8). There are 17 physiological human PARPs (9).

The two tankyrase proteins, tankyrase 1 (TNKS1; also known as PARP5A and ARTD5) and tankyrase 2 (TANK2; also known as PARP5B and ARTD6), belong to the PARP family (3). TNKS1 consists of an amino-terminal domain composed of homopolymeric stretches of His, Pro and Ser residues (the HPS domain), an ankyrin domain composed of 24 ankyrin repeats, a sterile $\alpha$ module (SAM) domain and a carboxy-terminal PARP catalytic domain $(10,11)$. TANK2 is associated with TNKS1 (10), but lacks an N-terminal HPS domain. The TANK2 ANK domain shares $83 \%$ identity with TNKS1, and the TANK2 SAM domain shares $74 \%$ identity with that of TNKS1 (10). The C-terminal PARP domain is a PARP polymerase and is highly conserved, with $94 \%$ identity (10). The ankyrin domain is implicated in protein-protein interactions (12), and the SAM domain is implicated in self-oligomerization (13). The HPS domain function is currently unknown.

Tankyrases interact with a number of target proteins and regulate cellular processes, including telomere maintenance, via telomere repeat binding factor 1 (TRF1) (10). Tankyrase-binding partners interact with TNKS1 using a 6-amino acid tankyrase-binding motif (RxxAxG, RxxPxG or RxxxxG) (14-17).

Tankyrases are involved in various cellular functions, including telomere maintenance (18), Wnt signaling (15), mitosis $(19-22)$, glucose metabolism $(23,24)$ and heritable disease cherubism $(14,25)$. Recent studies reported novel tankyrase binding partners, including phosphatase and tensin homolog (PTEN), peroxiredoxin II (PrxII), adenomatous polyposis coli 2 (APC2), angiomotins (AMOTs), abraxas brother 1 (ABRO1), cluster of differentiation 2 associated protein (CD2AP), peroxisomal biogenesis factor 14 (PEX14) and autophagy related 9A (ATG9A), as detailed in Table I (16,26-31). These data indicate novel tankyrase functionalities and provide novel insights for further investigations 
in numerous cellular responses. The present review focuses on novel tankyrase-binding partners and discusses recent data on tankyrase roles in cancer.

\section{Post-translational modification and tankyrase activity}

PARsylation and ubiquitination. Tankyrases catalyze post-translational modification of target proteins, which controls their stability $(15,32)$. TNKS1 PARsylates tankyrase target proteins, including TRF1, centrosomal P4.1-associated protein (CPAP), AXIN, PTEN and AMOTs $(15,16,21,32,33)$. The PARsylated protein is then recognized by the E3 ligase, and targeted for ubiquitination and proteasomal degradation $(15,16,26)$.

Tankyrase activity. Tankyrase activity is controlled by a variety of factors, including polo-like kinase-1 (PLK1) and mitogen-activated protein kinase (MAPK). TNKS1 is phosphorylated by PLK1, glycogen synthase kinase 3 (GSK3), and MAPK, although the precise functions of this modification are not clear (34-36). PLK1-mediated phosphorylation results in increased TNKS1 stability and telomeric PARP activity (34). GSK3-mediated phosphorylation of TNKS1 does not alter TNKS1 auto-PARsylation in vitro, and MAPK-mediated phosphorylation of tankyrase enhances the PARsylation activity of TNKS1 in vitro $(35,36)$. GDP-mannose 4, 6-dehydratase binds to TNKS1, inhibits tankyrase PARP activity in vitro and influences TNKS1 stability in vivo (37). Kang et al (26) demonstrated that TNKS1 is involved with the antioxidant enzyme PrxII. PrxII is essential for full TNKS1 activity, in order to maintain oncogenic $\beta$-catenin signaling in colorectal cancer (CRC). This study demonstrated the molecular mechanisms regulating tankyrase activity in CRC for the first time.

\section{Tankyrase and cancer}

Different biological tankyrase functions are relevant to cancer, including telomere maintenance, oncogenic pathways [Wnt, yes-associated protein (YAP) and AKT], mitosis, DNA repair and cell death, as depicted in Fig. 1.

Telomere maintenance. TNKS1 has been identified as an interaction partner of TRF1 $(18,38)$. TRF1 blocks the access of telomerase to telomeres $(18,32)$. TNKS1-mediated PARsylation of TRF1 releases TRF1 from telomeres, and the released TRF1 is degraded by the ubiquitin-proteasome pathway $(18,32)$. Telomere maintenance by telomerase allows continued proliferation of cancer cells and is considered as a promising target for anticancer strategies. TNKS1 controls telomerase inhibition in human cancer cells and is a potential telomere-directed anticancer target $(39,40)$. Telomere-directed inhibitors result in progressive telomere shortening, with no acute cytotoxicity, and combination with tankyrase inhibitors has been proposed $(39,40)$. Dual inhibition of TNKS1 and telomerase has demonstrated a synergistic effect in lung and gastric cancer cell lines $(41,42)$. Furthermore, the combination of tankyrase and telomerase inhibition promotes human lung adenocarcinoma cell apoptosis and inhibits proliferation (41). These observations indicate that co-inhibition of telomerase and tankyrase may be an effective strategy for the treatment of lung cancer in humans.
Oncogenic pathways. Tankyrases have been implicated in oncogenic pathways (Wnt, YAP and AKT) $(15,16,43)$.

Wnt signaling. The Wnt signal transduction pathway regulates numerous biological processes in diseases such as in cancer (44). AXIN is the key effector in the Wnt pathway and has been identified as a tumor suppressor. Tankyrases target AXIN for degradation, whereas tankyrase inhibitors generally stabilize it $(44,45)$. The Wnt pathway regulates proteolysis of the downstream effector $\beta$-catenin with the $\beta$-catenin destruction complex, which includes adenomatous polyposis coli (APC), AXIN and GSK3 $\beta$ (45). TNKS1-mediated AXIN PARsylation induces AXIN degradation with the ubiquitin-proteasome pathway, and the ensuing AXIN degradation triggers disruption of the $\beta$-catenin destruction complex (15). Released $\beta$-catenin translocates into the nucleus and switches on Wnt-dependent transcription (44).

The tumor suppressor APC scaffolds the $\beta$-catenin destruction complex (45). APC is mutated in $>80 \%$ of CRC cases (46). Therefore, due to tankyrases regulating Wnt signaling, tankyrase inhibitors may be promising therapeutic targets for CRC. TNKS1 inhibition suppresses Wnt signaling and tumor growth in APC-mutant colorectal tumors $(15,47,48)$, and increases chemosensitivity in colon cancer cell lines (49). Due to the Wnt pathway being involved in lung cancer $(50,51)$, antagonizing the Wnt pathway through tankyrase inhibition may be effective against lung cancer, and there is evidence for tankyrases as antineoplastic targets in lung cancer $(52,53)$.

Croy et al (27) indicated that the fly APC homolog APC2 may be a tankyrase substrate and that tankyrases regulate destruction complex activity, providing additional insight into tankyrase inhibition as a potential Wnt-pathway cancer therapy.

YAP signaling. The Hippo pathway controls tissue homeostasis and organ size $(54,55)$. YAP has been identified as an oncoprotein and the key effector in the Hippo pathway (54-58). YAP signaling has also been demonstrated to be involved in human cancer types (56-58). AMOTs are negative YAP regulators (33), and recent studies indicated that tankyrase inhibition suppresses YAP oncogenic activity by stabilizing AMOTs through inhibiting their tankyrase RNF146 axis-mediated degradation $(28,59)$. These results indicate a potential opportunity for cancer therapy. Lin et al (43) demonstrated that YAP signaling is involved in drug resistance, including with RAFand MAPK-targeted cancer therapy. Wang et al (59) reported that tankyrase inhibition enhances epidermal growth factor receptor (EGFR) growth inhibition in non-small cell lung cancer (NSCLC). These data indicate that tankyrase inhibition could be an effective approach to overcome drug resistance for combinatorial cancer therapy.

AKT signaling. PTEN is an important tumor suppressor, and PTEN mutations have been associated with a number of cancer types $(60,61)$ and Cowden syndrome (62). Li et al $(16)$ identified PTEN as a tankyrase-binding protein. PTEN stabilization by tankyrase inhibition induces downregulation of AKT phosphorylation, suppressing cell proliferation and tumor growth. These data support the therapeutic 
Table I. A summary of updated tankyrase-binding proteins.

\begin{tabular}{lll}
\hline Authors, year & Novel tankyrase-binding partners & Tankyrase-binding motif \\
\hline Li et al, 2015 & PTEN & RXXXDG \\
Kang et al, 2017 & PrXII & N.D. \\
Croy et al, 2016 & APC2 & RXXXXG \\
Wang et al, 2015 & AMOTs & RXXPXG \\
Tripathi and Smith, 2017 & ABRO1 & RXXAXG \\
Kuusela et al, 2016 & CD2AP & N.D. \\
Li et al, 2017 & PEX14 & RXXXXG, RXXXDG \\
Li et al, 2017 & ATG9A & RXXXXG
\end{tabular}

N.D., not determined; PTEN, phosphatase and tensin homolog; AMOTs, angiomotins; CD2AP, cluster of differentiation 2-associated protein; ABRO1, abraxas brother 1; APC2, adenomatous polyposis coli 2; PEX14, peroxisomal biogenesis factor 14; PrxII, peroxiredoxin II; ATG9A, autophagy related $9 \mathrm{~A}$.

potential of tankyrase inhibitors targeting the AKT oncogenic pathway.

Mitosis regulation. TNKS1 is required to resolve sister telomeres during mitosis. Sister chromatid cohesion holds sister chromatids together from their $\mathrm{S}$ phase replication until their mitosis separation $(22,29)$. Cohesion requires a multi-protein complex comprising structural maintenance of chromosomes protein $(\mathrm{Smc}) 1, \mathrm{Smc} 3$, sister chromatid cohesion protein (Scc)1 and Scc3 $(63,64)$. In the absence of TNKS1, cohesion is removed from arms and centromeres, but sister telomeres remain associated, indicating persistent cohesion, and this persistent telomere cohesion by TNKS1 inhibition during mitosis induces a delay in anaphase progression (22). Tripathi and Smith (29) demonstrated that the mechanism of cell cycle-regulated K63-ubiquitination of tankyrase controls sister telomere resolution timing.

TNKS1 colocalizes with the nuclear mitotic apparatus (NuMA) protein and PARsylates NuMA in mitosis (20), and TNKS1-depleted cells exhibit defects in mitotic spindle assembly and structure (19), indicating that TNKS1 is required for normal spindle formation. TNKS1 also localizes at the centrosomes, promotes centrosome maturation, interacts with CPAP, PARsylates CPAP, and regulates CPAP protein stability and function at centrosomes across the cell cycle (21). Miki PARsylation by TNKS1 promotes centrosome maturation (65); therefore, CPAP and Miki may have a general role in centrosome function. Abnormal centrosomes are involved in cancer and contribute to chromosome missegregation and aneuploidy, thereby promoting malignant progression (66-69). Korzeniewski et al (70) indicated centrosomes as a potential target for cancer therapy.

DNA repair. The DNA-dependent protein kinase (DNA-PK) is a critical component of non-homologous end joining-mediated DNA repair mechanisms (71). DNA-PKcs, a catalytic subunit of DNA-PK, exists in a PARsylated state in vitro and in vivo $(72,73)$. TNKS1 regulates DNA repair via PARsylation-mediated stabilization of DNA-PK and suppression of telomere-associated sister chromatid exchange (74).
Tankyrases bind to mediator of DNA damage checkpoint protein 1 and promote homologous recombination and checkpoint activation in response to double-strand breaks (DSBs) (75). Therefore, tankyrases have a direct role in DSB repair. DNA-PK is involved in tumor-associated processes, including genomic stability, hypoxia, metabolism, inflammatory response and transcription (71), which indicates that DNA-PK may be a potential target for cancer therapy.

Cell death. Tankyrase inhibition blocks proliferation and promotes cell apoptosis in neuroblastoma (NB) cell lines; therefore, tankyrases are a potential target for NB (17).

\section{Tankyrase inhibitors}

Numerous studies have reported the importance and utility of tankyrase inhibitors as cancer therapeutics (15,47,48,52,76-80). Consequently, a number of tankyrase inhibitors with promising therapeutic effects have been developed, including XAV939, IWR-1, G007-LK, JW55, AZ1366, JW 74 and NVP-TNKS656 (15,47,48,52,76-80) (Table II).

Tankyrase inhibition suppresses Wnt signaling and tumor growth in APC-mutant colorectal tumors $(15,47,48)$. Wu et al (49) demonstrated that the tankyrase inhibitor XAV939 increased chemosensitivity in colon cancer cell lines via inhibition of the Wnt signaling pathway. Lau et al (47) indicated that the tankyrase inhibitor G007-LK suppressed APC-mutant colorectal tumor growth. Mashima et al (81) reported that mechanistic target of rapamycin (mTOR) signaling conferred resistance to tankyrase inhibitors in Wnt-driven CRC, indicating that co-inhibition of tankyrase and mTOR may be an effective therapeutic approach for CRC.

The Wnt pathway is also involved in lung cancer $(50,51)$, and therefore antagonizing the Wnt pathway through tankyrase inhibition could be effective against lung cancer. Casás-Selves et al (53) and Busch et al (52) demonstrated tankyrase to be an antineoplastic target in lung cancer. Wang et al (59) indicated that the tankyrase inhibitor NVP-TNKS656 sensitized lung cancer cells to the EGFR inhibitor erlotinib. Busch et al (52) screened A375 melanoma 

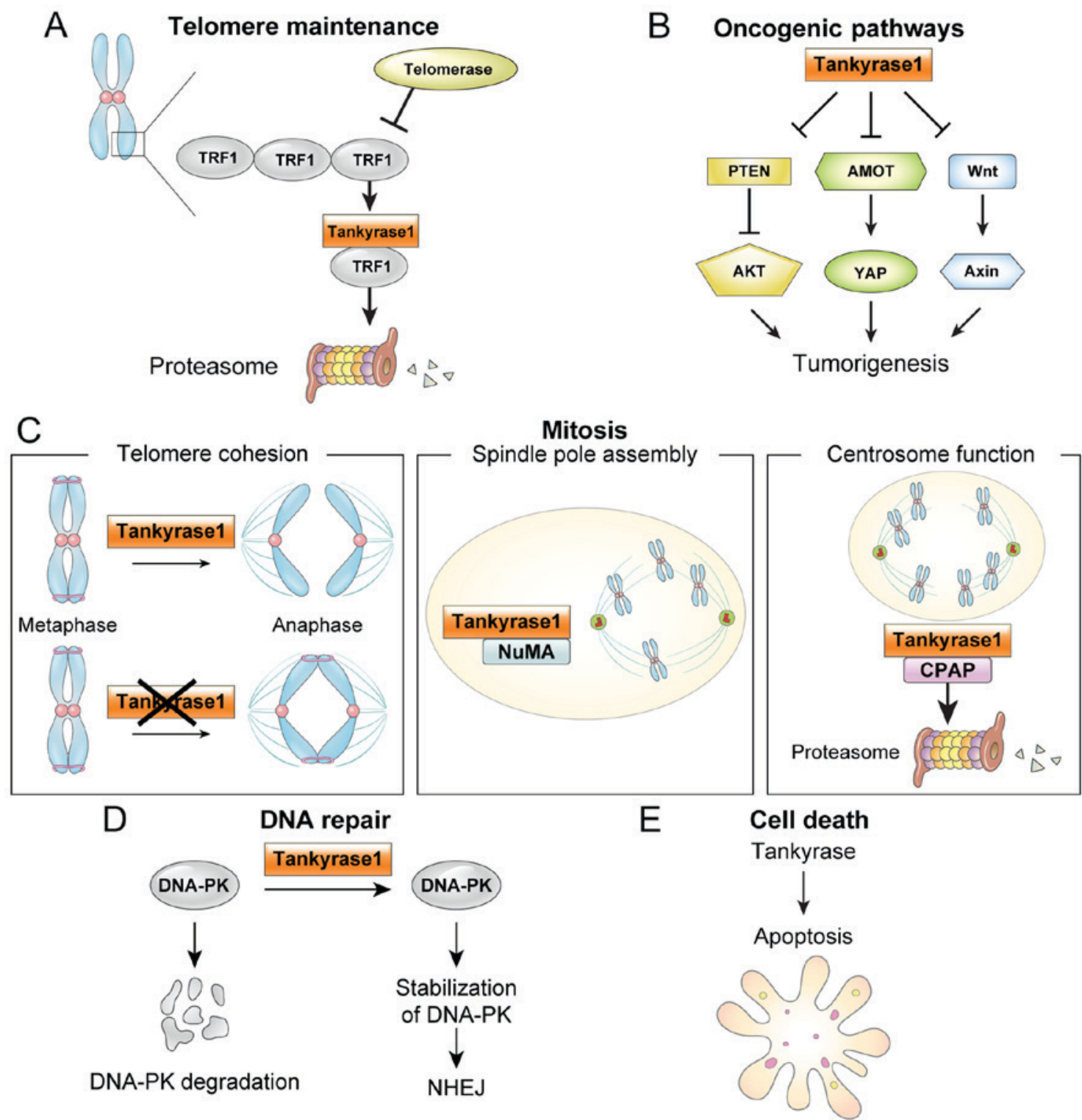

Figure 1. Tankyrase function in cancer. (A) ADP-ribosylation of TRF1 by tankyrase 1 releases TRF1 from telomeres, and the released TRF1 is degraded by the ubiquitin-proteasome pathway. Thus, telomere maintenance by telomerase allows continued proliferation. (B) Oncogenic pathways. Tankyrases are implicated in a number oncogenic pathways, including Wnt, YAP and AKT. (C) Mitosis. Tankyrase 1 has multiple functions in mitosis, including: i) required to resolve sister telomeres during mitosis; ii) localized to mitotic spindle poles during mitosis, where NuMA PARsylation is required for normal spindle formation and iii) regulates CPAP protein stability and function by its PARsylation. (D) DNA repair. Tankyrase 1 stabilizes the NHEJ protein DNA-PK. (E) Apoptosis. Tankyrases are involved in apoptosis, although the mechanism is unclear. TRF1, telomere repeat binding factor 1; PTEN, phosphatase and tensin homolog; YAP, yes-associated protein; AMOT, angiomotins; NuMA, nuclear mitotic apparatus; CPAP, centrosomal P4.1-associated protein; DNA-PK, DNA-dependent protein kinase; NHEJ, non-homologous end joining.

cells and identified WIKI4, a small molecule inhibitor of Wnt $/ \beta$-catenin signaling.

Co-localization of the transcription factor forkhead box $\mathrm{O} 3$ and $\beta$-catenin in the nucleus mediates progression and metastasis in CRC upon phosphoinositide 3-kinase (PI3K) or AKT inhibition $(36,82)$. Co-exposure to AKT or PI3K inhibitors and the tankyrase inhibitor XAV939 impairs metastasis. Arqués et al (76) demonstrated that the tankyrase inhibitor NVP-TNKS656 blocked the Wnt/ $\beta$-catenin pathway, overcoming resistance to PI3K and AKT inhibitors in CRC.

Thomson et al (83) characterized a novel tankyrase inhibitor, tetrazoloquinoxaline 41 , and indicated that it inhibited growth in tumor-derived cell lines, providing a potential cancer therapy.

\section{Novel tankyrase binding partners}

Numerous tankyrase binding partners have been reviewed (2), and a number of studies have recently reported novel tankyrase binding partners (16,26-31). This section reviews a number of these novel tankyrase binding partners, including PTEN, PrxII, APC2, AMOTs, ABRO1, CD2AP, PEX14 and ATG9A (Table I).

$P T E N$. PTEN has been characterized as a tumour suppressor, and PTEN mutations have been reported in cancers $(60,61)$ and Cowden syndrome (62). Li et al (16) identified PTEN as a tankyrase-binding protein containing a RXXXDG tankyrase-binding motif (RYQEDG). Tankyrases interact 
Table II. Tankyrase inhibitors as therapeutic targets for cancer.

\begin{tabular}{|c|c|c|c|}
\hline Author, year & Tankyrase inhibitors & Cancer type & (Refs.) \\
\hline Stratford et al, 2014 & JW 74 & Osteosarcoma & $(79)$ \\
\hline Tian et al, 2013 & & NB & $(17)$ \\
\hline Huang et al,2009 & XAV939 & $\mathrm{CRC}$ & $(15)$ \\
\hline Busch et al, 2013 & & Lung cancer & $(52)$ \\
\hline Bao et al, 2012 & & Breast cancer & $(77)$ \\
\hline Quackenbush et al,2016 & AZ1366 & $\mathrm{CRC}$ & $(78)$ \\
\hline Busch et al, 2013 & IWR-1 & Lung cancer & $(52)$ \\
\hline Lau et al, 2013 & G007-LK & $\mathrm{CRC}$ & $(47)$ \\
\hline Waaler et al, 2012 & JW55 & $\mathrm{CRC}$ & $(48)$ \\
\hline Arqués et al, 2016 & NVP-TNKS656 & NSCLC & $(76)$ \\
\hline Wang et al, 2016 & & & $(59)$ \\
\hline
\end{tabular}

NB, neuroblastoma; CRC, colorectal cancer; NSCLC, non-small cell lung cancer.

with and PARsylate PTEN. PARsylated PTEN promotes PTEN degradation through E3 ligase RNF146 (16).

PTEN stabilization by tankyrase inhibition induces downregulation of AKT phosphorylation, suppressing cell proliferation and tumor growth (16). These data indicate a therapeutic potential for tankyrase inhibitors in cancer, targeting the AKT oncogenic pathway.

PrxII. Kang et al (26) demonstrated that TNKS1 is involved with PrxII, and PrxII is essential for full TNKS1 activity to maintain oncogenic $\beta$-catenin signaling in CRC. In addition, it was indicated that the TNKS1 zinc-binding motif is essential for PARP activity and is protected from oxidative inactivation by PrxII. Furthermore, $\mathrm{H}_{2} \mathrm{O}_{2}$-dependent inactivation of TNKS1 PARP activity in the absence of PrxII enhances AXIN-dependent $\beta$-catenin degradation in APC-mutant CRC cells (26). These results indicate that PrxII inhibition may exert therapeutic effects on APC-mutant CRC cells.

$A P C 2$. Of all colon cancer cases, $>80 \%$ are initiated by truncating mutations in the tumor suppressor APC (46). The Wnt pathway regulates proteolysis of the downstream effector $\beta$-catenin via the $\beta$-catenin destruction complex, including APC, AXIN and GSK3 $\beta$ (45). Croy et al (27) identified APC2, a fly APC homolog, as a tankyrase binding partner and substrate. This previous study indicated that tankyrases regulate the activity of the $\beta$-catenin destruction complex through AXIN and APC2 ribosylation, supporting the therapeutic value of tankyrase inhibition as a Wnt-pathway cancer therapy.

Angiomotin family of proteins. AMOTs are negative YAP regulators (33), and tankyrase inhibition suppresses YAP oncogenic activity by stabilizing AMOT family proteins $(28,59)$. Tankyrases bind to AMOTs, and tankyrase-mediated PARsylation induces their degradation through E3 ligase RNF146 $(28,59)$. These observations highlight the therapeutic potential of tankyrase inhibitors in cancer, targeting the YAP oncogenic pathway. YAP signaling has been associated with drug resistance in cancer types, including lung cancer, and hence YAP signaling inhibition is important to overcome drug resistance (84). Tankyrase inhibition enhances EGFR inhibitor growth inhibition in lung cancer cells via AMOT stabilization and YAP signaling inhibition (59). Thus, tankyrase inhibition may be an effective approach to overcoming drug resistance for combinatorial cancer therapy.

ABRO1. During the S phase, DNA must not only be replicated, but also newly synthesized DNA molecules must also be connected with each other (85). This sister chromatid cohesion is essential for chromosome segregation during mitosis. Canudas and Smith (86) demonstrated that premature resolution of telomere cohesion between sister telomeres induced sister telomere loss. Resolution at telomeres requires TNKS1, but TNKS1 mechanisms in timely resolution of sister telomere cohesion are poorly understood. Tripathi and Smith (29) identified ABRO1, the scaffold subunit of the BRCC36 deubiquitinating enzyme (BRISC DUB) and demonstrated that sister telomere resolution timing was ensured through cell cycle-regulated ubiquitination of TNKS1 by RNF8 ligase and the BRISC DUB. Perturbation of this regulation results in persistent unresolved cohesion in mitosis or premature loss of cohesion in the $\mathrm{S}$ phase, indicating that a cell cycle-regulated post-translational modification controls sister telomere cohesion timing to ensure genome integrity.

CD2 associated protein. The adapter protein CD2AP is essential for kidney ultrafiltration and is expressed primarily by podocytes in the kidney. Podocyte damage results in numerous glomerular diseases, including nephritic syndrome and nephrotic syndrome (87). Kuusela et al (30) demonstrated that tankyrases interact with CD2AP, and CD2AP is a negative tankyrase regulator. Tankyrase inhibition in the absence of CD2AP increases kidney damage, which indicates that tankyrases are essential for maintaining normal kidney function (87). 
PEX14 and ATG9A. Li et al (31) investigated the tankyrase protein interaction network through proteomic analysis and identified $>100$ high-confidence interacting proteins for tankyrases. In particular, they demonstrated that TNKS1 and TANK2 bind to the peroxisome protein PEX14 and localize on peroxisomes. Overexpression of TNKS1 or TANK2 decreases peroxisome number or size, indicating that TNKS1 and TANK2 promote pexophagy. This study also demonstrated that tankyrases associate with the autophagy-associated protein ATG9A. Additionally, they indicated that tankyrase may associate PEX14 with ATG9A to promote pexophagy. Further experimentation is required to confirm the detailed mechanism. This study provides insights into further cellular localizations and functions.

\section{Conclusions}

Tankyrases have been implicated in a variety of cellular functions and are important therapeutic targets; however, the details of tankyrase functions and molecular mechanisms remain unclear. Novel tankyrase binding partners, including PTEN, AMOTs, CD2AP, APC2, ABRO1, PrxII, PEX14 and ATG9A have been recently reported, and these proteins will provide novel insights to understand the functions and mechanisms of tankyrase.

A number of tankyrase substrates are tumor suppressors, including AXIN, PTEN and AMOTs. Due to tankyrase inhibitors targeting different oncogenic pathways, including WNT, $\mathrm{AKT}$ and YAP, tankyrases may be effective targets for cancer therapy.

\section{Acknowledgements}

Not applicable.

\section{Funding}

This research was supported by the National Research Foundation of Korea (grant no. NRF-2015R1C1A1A02037631).

\section{Availability of data and materials}

Not applicable.

\section{Authors' contributions}

MKK designed the present review, collected information and wrote the manuscript.

\section{Ethics approval and consent to participate}

Not applicable.

\section{Patient consent for publication}

Not applicable.

\section{Competing interests}

The author declares that they have no competing interests.

\section{References}

1. Bürkle A: Poly(ADP-ribose). The most elaborate metabolite of NAD+. FEBS J 272: 4576-4589, 2005

2. Haikarainen T, Krauss S and Lehtio L: Tankyrases: Structure, function and therapeutic implications in cancer. Curr Pharm Des 20: 6472-6488, 2014.

3. Riffell JL, Lord CJ and Ashworth A: Tankyrase-targeted therapeutics: Expanding opportunities in the PARP family. Nat Rev Drug Discov 11: 923-936, 2012.

4. Malanga $\mathrm{M}$ and Althaus FR: The role of poly(ADP-ribose) in the DNA damage signaling network. Biochem Cell Biol 83: 354-364, 2005.

5. Luo X and Kraus WL: On PAR with PARP: Cellular stress signaling through poly(ADP-ribose) and PARP-1. Genes Dev 26: 417-432, 2012.

6. Kraus WL and Lis JT: PARP goes transcription. Cell 113: 677-683, 2003.

7. Yeh TY, Sbodio JI, Tsun ZY, Luo B and Chi NW: Insulin-stimulated exocytosis of GLUT4 is enhanced by IRAP and its partner tankyrase. Biochem J 402: 279-290, 2007.

8. Beneke S and Bürkle A: Poly(ADP-ribosyl)ation in mammalian ageing. Nucleic Acids Res 35: 7456-7465, 2007.

9. Otto H, Reche PA, Bazan F, Dittmar K, Haag F and Koch-Nolte F: In silico characterization of the family of PARP-like poly(ADP-ribosyl) transferases (pARTs). BMC Genomics 6: 139, 2005.

10. Hsiao SJ and Smith S: Tankyrase function at telomeres, spindle poles, and beyond. Biochimie 90: 83-92, 2008.

11. Smith S, Giriat I, Schmitt A and de Lange T: Tankyrase, a poly(ADP-ribose) polymerase at human telomeres. Science 282: 1484-1487, 1998.

12. Seimiya H and Smith S: The telomeric poly(ADP-ribose) polymerase, tankyrase 1 , contains multiple binding sites for telomeric repeat binding factor 1 (TRF1) and a novel acceptor, 182-kDa tankyrase-binding protein (TAB182). J Biol Chem 277: 14116-14126, 2002.

13. De Rycker M and Price CM: Tankyrase polymerization is controlled by its sterile alpha motif and poly(ADP-ribose) polymerase domains. Mol Cell Biol 24: 9802-9812, 2004.

14. Guettler S, LaRose J, Petsalaki E, Gish G, Scotter A, Pawson T, Rottapel R and Sicheri F: Structural basis and sequence rules for substrate recognition by Tankyrase explain the basis for cherubism disease. Cell 147: 1340-1354, 2011.

15. Huang SM, Mishina YM, Liu S, Cheung A, Stegmeier F, Michaud GA, Charlat O, Wiellette E, Zhang Y, Wiessner S, et al: Tankyrase inhibition stabilizes axin and antagonizes Wnt signalling. Nature 461: 614-620, 2009.

16. Li N, Zhang Y, Han X, Liang K, Wang J, Feng L, Wang W, Songyang Z, Lin C, Yang L, et al: Poly-ADP ribosylation of PTEN by tankyrases promotes PTEN degradation and tumor growth. Genes Dev 29: 157-170, 2015.

17. Tian XH, Hou WJ, Fang Y, Fan J, Tong H, Bai SL, Chen Q, Xu H and Li Y: XAV939, a tankyrase 1 inhibitior, promotes cell apoptosis in neuroblastoma cell lines by inhibiting Wnt/ $\beta$-catenin signaling pathway. J Exp Clin Cancer Res 32: 100, 2013.

18. Smith S and de Lange T: Tankyrase promotes telomere elongation in human cells. Curr Biol 10: 1299-1302, 2000.

19. Chang P, Coughlin M and Mitchison TJ: Tankyrase-1 polymerization of poly(ADP-ribose) is required for spindle structure and function. Nat Cell Biol 7: 1133-1139, 2005.

20. Chang W, Dynek JN and Smith S: NuMA is a major acceptor of poly(ADP-ribosyl)ation by tankyrase 1 in mitosis. Biochem J 391: 177-184, 2005.

21. Kim MK, Dudognon C and Smith S: Tankyrase 1 regulates centrosome function by controlling CPAP stability. EMBO Rep 13: 724-732, 2012.

22. Kim MK and Smith S: Persistent telomere cohesion triggers a prolonged anaphase. Mol Biol Cell 25: 30-40, 2014.

23. Guo HL, Zhang C, Liu Q, Li Q, Lian G, Wu D, Li X, Zhang W, Shen Y, Ye Z, et al: The Axin/TNKS complex interacts with KIF3A and is required for insulin-stimulated GLUT4 translocation. Cell Res 22: 1246-1257, 2012.

24. Yeh TY, Sbodio JI and Chi NW: Mitotic phosphorylation of tankyrase, a PARP that promotes spindle assembly, by GSK3. Biochem Biophys Res Commun 350: 574-579, 2006.

25. Levaot N, Voytyuk O, Dimitriou I, Sircoulomb F, Chandrakumar A, Deckert M,Krzyzanowski PM, Scotter A, Gu S, Janmohamed S, et al: Loss of Tankyrase-mediated destruction of 3BP2 is the underlying pathogenic mechanism of cherubism. Cell 147: 1324-1339, 2011. 
26. Kang DH, Lee DJ, Lee S, Lee SY, Jun Y, Kim Y, Kim Y, Lee JS, Lee DK, Lee S, et al: Interaction of tankyrase and peroxiredoxin II is indispensable for the survival of colorectal cancer cells. Nat Commun 8: 40, 2017.

27. Croy HE, Fuller CN, Giannotti J, Robinson P, Foley AV, Yamulla RJ, Cosgriff S, Greaves BD, von Kleeck RA, An $\mathrm{HH}$, et al: The poly(ADP-ribose) polymerase enzyme tankyrase antagonizes activity of the $\beta$-catenin destruction complex through ADP-ribosylation of axin and APC2. J Biol Chem 291: 12747-12760, 2016.

28. Wang W, Li N, Li X, Tran MK, Han X and Chen J: Tankyrase inhibitors target YAP by stabilizing angiomotin family proteins. Cell Rep 13: 524-532, 2015.

29. Tripathi E and Smith S: Cell cycle-regulated ubiquitination of tankyrase 1 by RNF8 and ABRO1/BRCC 36 controls the timing of sister telomere resolution. EMBO J 36: 503-519, 2017.

30. Kuusela S, Wang H, Wasik AA, Suleiman H and Lehtonen S: Tankyrase inhibition aggravates kidney injury in the absence of CD2AP. Cell Death Dis 7: e2302, 2016.

31. Li X, Han H, Zhou MT, Yang B, Ta AP, Li N, Chen J and Wang W: Proteomic analysis of the human tankyrase protein interaction network reveals its role in pexophagy. Cell Rep 20: 737-749, 2017

32. Chang W, Dynek JN and Smith S: TRF1 is degraded by ubiquitin-mediated proteolysis after release from telomeres. Genes Dev 17: 1328-1333, 2003.

33. Wang W, Huang J and Chen J: Angiomotin-like proteins associate with and negatively regulate YAP1. J Biol Chem 286: 4364-4370, 2011

34. Ha GH, Kim HS, Go H, Lee H, Seimiya H, Chung DH and Lee CW: Tankyrase-1 function at telomeres and during mitosis is regulated by Polo-like kinase-1-mediated phosphorylation. Cell Death Differ 19: 321-332, 2012.

35. Chi NW and Lodish HF: Tankyrase is a golgi-associated mitogen-activated protein kinase substrate that interacts with IRAP in GLUT4 vesicles. J Biol Chem 275: 38437-38444, 2000.

36. Yan Y and Lackner MR: FOXO3a and $\beta$-catenin co-localization: Double trouble in colon cancer? Nat Med 18: 854-856, 2012.

37. Bisht KK, Dudognon C, Chang WG, Sokol ES, Ramirez A and Smith S: GDP-mannose-4,6-dehydratase is a cytosolic partner of tankyrase 1 that inhibits its poly(ADP-ribose) polymerase activity. Mol Cell Biol 32: 3044-3053, 2012.

38. Kaminker PG, Kim SH, Taylor RD, Zebarjadian Y, Funk WD, Morin GB, Yaswen $\mathrm{P}$ and Campisi J: TANK2, a new TRF1-associated poly(ADP-ribose) polymerase, causes rapid induction of cell death upon overexpression. J Biol Chem 276 : 35891-35899, 2001.

39. Cerone MA, Burgess DJ, Naceur-Lombardelli C, Lord CJ and Ashworth A: High-throughput RNAi screening reveals novel regulators of telomerase. Cancer Res 71: 3328-3340, 2011

40. Seimiya H, Muramatsu Y, Ohishi T and Tsuruo T: Tankyrase 1 as a target for telomere-directed molecular cancer therapeutics Cancer Cell 7: 25-37, 2005

41. Lu H, Lei Z, Lu Z, Lu Q, Lu C, Chen W, Wang C, Tang Q and Kong Q: Silencing tankyrase and telomerase promotes A549 human lung adenocarcinoma cell apoptosis and inhibits proliferation. Oncol Rep 30: 1745-1752, 2013.

42. Zhang H, Yang MH, Zhao JJ, Chen L, Yu ST, Tang XD, Fang DC and Yang SM: Inhibition of tankyrase 1 in human gastric cancer cells enhances telomere shortening by telomerase inhibitors. Oncol Rep 24: 1059-1065, 2010.

43. Lin L, Sabnis AJ, Chan E, Olivas V, Cade L, Pazarentzos E, Asthana S, Neel D, Yan JJ, Lu X, et al: The Hippo effector YAP promotes resistance to RAF- and MEK-targeted cancer therapies. Nat Genet 47: 250-256, 2015.

44. Clevers H: Wnt/beta-catenin signaling in development and disease. Cell 127: 469-480, 2006.

45. Rubinfeld B, Albert I, Porfiri E, Fiol C, Munemitsu S and Polakis P: Binding of GSK3beta to the APC-beta-catenin complex and regulation of complex assembly. Science 272: 1023-1026, 1996.

46. Cancer Genome Atlas Network: Comprehensive molecular characterization of human colon and rectal cancer. Nature 487: 330-337, 2012.

47. Lau T, Chan E, Callow M, Waaler J, Boggs J, Blake RA, Magnuson S, Sambrone A, Schutten M, Firestein R, et al: A novel tankyrase small-molecule inhibitor suppresses APC mutation-driven colorectal tumor growth. Cancer Res 73: 3132-3144, 2013.

48. Waaler J, Machon O, Tumova L, Dinh H, Korinek V, Wilson SR, Paulsen JE, Pedersen NM, Eide TJ, Machonova O, et al: A nove tankyrase inhibitor decreases canonical Wnt signaling in colon carcinoma cells and reduces tumor growth in conditional APC mutant mice. Cancer Res 72: 2822-2832, 2012.
49. Wu X, Luo F, Li J, Zhong X and Liu K: Tankyrase 1 inhibitior XAV939 increases chemosensitivity in colon cancer cell lines via inhibition of the Wnt signaling pathway. Int $\mathrm{J}$ Oncol 48: 1333-1340, 2016

50. Nguyen DX, Chiang AC, Zhang XH, Kim JY, Kris MG, Ladanyi M, Gerald WL and Massagué J: WNT/TCF signaling through LEF1 and HOXB9 mediates lung adenocarcinoma metastasis. Cell 138: 51-62, 2009.

51. Pacheco-Pinedo EC, Durham AC, Stewart KM, Goss AM, Lu MM, Demayo FJ and Morrisey EE: Wnt/ $\beta$-catenin signaling accelerates mouse lung tumorigenesis by imposing an embryonic distal progenitor phenotype on lung epithelium. J Clin Invest 121: 1935-1945, 2011.

52. Busch AM, Johnson KC, Stan RV, Sanglikar A, Ahmed Y, Dmitrovsky E and Freemantle SJ: Evidence for tankyrases as antineoplastic targets in lung cancer. BMC Cancer 13: 211, 2013.

53. Casás-Selves M, Kim J, Zhang Z, Helfrich BA, Gao D, Porter CC, Scarborough HA, Bunn PA Jr, Chan DC, Tan AC and DeGregori J: Tankyrase and the canonical Wnt pathway protect lung cancer cells from EGFR inhibition. Cancer Res 72: 4154-4164, 2012

54. Halder G and Johnson RL: Hippo signaling: Growth control and beyond. Development 138: 9-22, 2011.

55. Zhao B, Li L, Lei Q and Guan KL: The Hippo-YAP pathway in organ size control and tumorigenesis: An updated version. Genes Dev 24: 862-874, 2010.

56. Dong J, Feldmann G, Huang J, Wu S, Zhang N, Comerford SA, Gayyed MF, Anders RA, Maitra A and Pan D: Elucidation of a universal size-control mechanism in Drosophila and mammals. Cell 130: 1120-1133, 2007.

57. Harvey KF, Zhang X and Thomas DM: The Hippo pathway and human cancer. Nat Rev Cancer 13: 246-257, 2013.

58. Mo JS, Park HW and Guan KL: The Hippo signaling pathway in stem cell biology and cancer. EMBO Rep 15: 642-656, 2014.

59. Wang H, Lu B, Castillo J, Zhang Y, Yang Z, McAllister G, Lindeman A, Reece-Hoyes J, Tallarico J, Russ C, et al: Tankyrase inhibitor sensitizes lung cancer cells to Endothelial Growth Factor Receptor (EGFR) inhibition via stabilizing angiomotins and inhibiting YAP signaling. J Biol Chem 291: 15256-15266, 2016.

60. Li J, Yen C, Liaw D, Podsypanina K, Bose S, Wang SI, Puc J, Miliaresis C, Rodgers L, McCombie R, et al: PTEN, a putative protein tyrosine phosphatase gene mutated in human brain, breast, and prostate cancer. Science 275: 1943-1947, 1997.

61. Steck PA, Pershouse MA, Jasser SA, Yung WK, Lin H, Ligon AH, Langford LA, Baumgard ML, Hattier T, Davis T, et al: Identification of a candidate tumour suppressor gene, MMAC1, at chromosome $10 \mathrm{q} 23.3$ that is mutated in multiple advanced cancers. Nat Genet 15: 356-362, 1997.

62. Liaw D, Marsh DJ, Li J, Dahia PL, Wang SI, Zheng Z, Bose S, Call KM, Tsou HC, Peacocke M, et al: Germline mutations of the PTEN gene in Cowden disease, an inherited breast and thyroid cancer syndrome. Nat Genet 16: 64-67, 1997.

63. Losada A and Hirano T: Dynamic molecular linkers of the genome: The first decade of SMC proteins. Genes Dev 19: 1269-1287, 2005

64. Nasmyth $\mathrm{K}$ and Haering $\mathrm{CH}$ : The structure and function of SMC and kleisin complexes. Annu Rev Biochem 74: 595-648, 2005.

65. Ozaki Y, Matsui H, Asou H, Nagamachi A, Aki D, Honda H, Yasunaga S, Takihara Y, Yamamoto T, Izumi S, et al: Poly-ADP ribosylation of Miki by tankyrase-1 promotes centrosome maturation. Mol Cell 47: 694-706, 2012.

66. Boveri T: Concerning the origin of malignant tumours by Theodor Boveri. Translated and annotated by Henry Harris. J Cell Sci 121 (Suppl 1): S1-S84, 2008.

67. Duensing S and Münger K: Centrosome abnormalities, genomic instability and carcinogenic progression. Biochim Biophys Acta 1471: M81-M88, 2001

68. Ganem NJ, Godinho SA and Pellman D: A mechanism linking extra centrosomes to chromosomal instability. Nature 460: 278-282, 2009

69. Guerrero AA, Martínez-A C and van Wely KH: Merotelic attachments and non-homologous end joining are the basis of chromosomal instability. Cell Div 5: 13, 2010.

70. Korzeniewski N, Hohenfellner M and Duensing S: The centrosome as potential target for cancer therapy and prevention. Expert Opin Ther Targets 17: 43-52, 2013.

71. Goodwin JF and Knudsen KE: Beyond DNA repair: DNA-PK function in cancer. Cancer Discov 4: 1126-1139, 2014 
72. Gagné JP, Isabelle M, Lo KS, Bourassa S, Hendzel MJ, Dawson VL, Dawson TM and Poirier GG: Proteome-wide identification of poly(ADP-ribose) binding proteins and poly(ADP-ribose)-associated protein complexes. Nucleic Acids Res 36: 6959-6976, 2008.

73. Ruscetti T, Lehnert BE, Halbrook J, Le Trong H, Hoekstra MF, Chen DJ and Peterson SR: Stimulation of the DNA-dependent protein kinase by poly(ADP-ribose) polymerase. J Biol Chem 273: 14461-14467, 1998.

74. Dregalla RC, Zhou J, Idate RR, Battaglia CL, Liber HL and Bailey SM: Regulatory roles of tankyrase 1 at telomeres and in DNA repair: Suppression of T-SCE and stabilization of DNA-PKcs. Aging (Albany NY) 2: 691-708, 2010.

75. Nagy Z, Kalousi A, Furst A, Koch M, Fischer B and Soutoglou E: Tankyrase promote homologous recombination and check point activation in response to DSBs. PLoS Genet 12: e1005791, 2016.

76. Arqués O,Chicote I, Puig I, Tenbaum SP, Argilés G,Dienstmann R, Fernández N, Caratù G, Matito J, Silberschmidt D, et al: Tankyrase inhibition blocks Wnt/ $\beta$-catenin pathway and reverts resistance to $\mathrm{PI} 3 \mathrm{~K}$ and $\mathrm{AKT}$ inhibitors in the treatment of colorectal cancer. Clin Cancer Res 22: 644-656, 2016.

77. Bao R, Christova T, Song S, Angers S, Yan X and Attisano L: Inhibition of tankyrases induces Axin stabilization and blocks Wnt signalling in breast cancer cells. PLoS One 7: e48670, 2012

78. Quackenbush KS, Bagby S, Tai WM, Messersmith WA, Schreiber A, Greene J, Kim J, Wang G, Purkey A, Pitts TM, et al: The novel tankyrase inhibitor (AZ1366) enhances irinotecan activity in tumors that exhibit elevated tankyrase and irinotecan resistance. Oncotarget 7: 28273-28285, 2016

79. Stratford EW, Daffinrud J, Munthe E, Castro R, Waaler J, Krauss S and Myklebost O: The tankyrase-specific inhibitor JW74 affects cell cycle progression and induces apoptosis and differentiation in osteosarcoma cell lines. Cancer Med 3: 36-46, 2014.
80. Tian X, Hou W, Bai S, Fan J, Tong H and Xu H: XAV939 inhibits the stemness and migration of neuroblastoma cancer stem cells via repression of tankyrase 1. Int J Oncol 45: 121-128, 2014.

81. Mashima T, Taneda Y, Jang MK, Mizutani A, Muramatsu Y, Yoshida H, Sato A, Tanaka N, Sugimoto Y and Seimiya H: mTOR signaling mediates resistance to tankyrase inhibitors in Wnt-driven colorectal cancer. Oncotarget 8: 47902-47915, 2017.

82. Tenbaum SP, Ordóñez-Morán P, Puig I, Chicote I, Arqués O, Landolfi S, Fernández Y, Herance JR, Gispert JD, Mendizabal L, et al: $\beta$-catenin confers resistance to PI3K and AKT inhibitors and subverts FOXO3a to promote metastasis in colon cancer. Nat Med 18: 892-901, 2012.

83. Thomson DW, Wagner AJ, Bantscheff M, Benson RE, Dittus L, Duempelfeld B, Drewes G, Krause J, Moore JT, Mueller K, et al: Discovery of a highly selective tankyrase inhibitor displaying growth inhibition effects against a diverse range of tumor derived cell lines. J Med Chem 60: 5455-5471, 2017.

84. Keren-Paz A, Emmanuel R and Samuels Y: YAP and the drug resistance highway. Nat Genet 47: 193-194, 2015.

85. Peters JM and Nishiyama T: Sister chromatid cohesion. Cold Spring Harb Perspect Biol 4: pii: a011130, 2012.

86. Canudas S and Smith S: Differential regulation of telomere and centromere cohesion by the Scc3 homologues SA1 and SA2, respectively, in human cells. J Cell Biol 187: 165-173, 2009.

87. Matovinović MS: Podocyte injury in glomerular diseases. EJIFCC 20: 21-27, 2009.

(c) (7) (8) This work is licensed under a Creative Commons Attribution 4.0 International (CC BY-NC 4.0) License 